\title{
Portales educativos: la producción de materiales didácticos digitales ${ }^{1}$
}

\author{
Educational Portals: The Production of Digital Teaching Materials
}

\section{Carlos José González Ruiz}

Grupo de investigación Edullab. Laboratorio de Educación y Nuevas Tecnologías. Universidad de La Laguna cgonzalezruiz81@gmail.com

\section{Sebastián Martín Gómez}

Grupo de investigación Edullab. Laboratorio de Educación y Nuevas Tecnologías. Universidad de La Laguna smarting@ull.edu.es

\section{Ana Vega Navarro}

Grupo de investigación Edullab. Laboratorio de Educación y Nuevas Tecnologías. Universidad de La Laguna amvega@ull.edu.es

Fecha presentación: 13/03/2018 | Aceptación: 29/05/2018 |Publicación: 22/06/2018

\begin{abstract}
Resumen
El objetivo principal de este artículo es conocer cómo se organiza la producción de los materiales didácticos digitales de creación propia de portales institucionales de recursos educativos, tomando como referencia la perspectiva de sus principales responsables. En concreto, se analiza el caso del repositorio institucional de recursos de la Comunidad Autónoma de Canarias, Ecoescuela 2.0; y del portal de recursos educativos en abierto Procomún (Ministerio de Educación, Cultura y Deporte). Con este estudio se pretende dar respuesta a distintas cuestiones relacionadas con la autoría de los materiales, el proceso y desarrollo, así como los criterios que establecen los portales institucionales para la producción de los mismos. Para ello se opta por una metodología de investigación cualitativa, que trata de arrojar luz sobre los procesos existentes en cuanto al diseño y producción de dichos materiales digitales. Los resultados nos indican varias cuestiones: necesidad de seguir investigando sobre dicha cuestión, el cambio de autoría en la creación de los materiales didácticos, así como la inclusión del profesorado en el proceso de creación y desarrollo de los mismos.
\end{abstract}

Palabras clave: portal educativo; portal institucional; material didáctico digital; creación de recursos; escuela digital

\begin{abstract}
Resum
L'objectiu principal d'aquest article és conèixer com s'organitza la producció dels materials didàctics digitals de creació pròpia de portals institucionals de recursos educatius, prenent com a referència la perspectiva dels seus principals responsables. En concret, s'analitza el cas del repositori institucional de recursos de la Comunitat Autònoma de Canàries, Ecoescola 2.0; i del portal de recursos educatius en obert Comuns (Ministeri d'Educació, Cultura i Esport). Amb aquest estudi es pretén donar resposta a diferents qüestions relacionades amb l'autoria dels materials, el procés i desenvolupament, així com els criteris que estableixen els portals institucionals per a la producció dels mateixos. Per això s'opta per una metodologia d'investigació qualitativa, que tracta de llançar llum sobre els processos existents pel que fa al disseny i producció d'aquests materials digitals. Els resultats ens indiquen diverses qüestions: necessitat de seguir investigant sobre aquesta qüestió, el canvi d'autoria en la creació dels materials didàctics, així com la inclusió del professorat en el procés de creació i desenvolupament dels mateixos.
\end{abstract}

Paraules clau: portal educatiu; portal institucional; material didàctic digital; creació de recursos; escola digital

\footnotetext{
1 Este artículo es resultado del proyecto de investigación titulado "La escuela de la sociedad digital: análisis y propuestas para la producción y uso de los contenidos digitales educativos" (EDU2015-64593-R). Financiado por el Programa Estatal de I+D+i Orientada a los Retos de la Sociedad convocado por el Gobierno de España.
} 
González Ruiz, Carlos José; Martín Gómez, Sebastián y Vega Navarro, Ana (2018). "Portales educativos: la producción de materiales didácticos digitales". @tic revista d'innovació educativa, 20, 89-97.

\section{Abstract}

The main objective of this article is to know how the production of the digital didactic materials created by institutional portals of educational resources is organized, taking as a reference the perspective of its main responsible. In particular, the case of the institutional repository of resources of the Autonomous Community of the Canary Islands, Ecoescuela 2.0; and the portal of open educational resources Procomún (Ministry of Education, Culture and Sports). This study aims to answer different questions related to the authorship of the materials, the process and development, as well as the criteria established by the institutional portals for their production. For this purpose, a qualitative research methodology is chosen, which tries to shed light on the existing processes regarding the design and production of said digital materials. The results indicate several issues: the need to continue researching this issue, the change of authorship in the creation of teaching materials, as well as the inclusion of teachers in the process of creating and developing them.

Key Words: educational portal; institutional portal; digital didactic material; creation of resources; digital school

\section{Introducción}

Teniendo en cuenta la sociedad en la que actualmente vivimos, donde la tecnología está a la orden del día y los dispositivos tecnológicos ocupan una gran parte del tiempo de jóvenes y mayores, no es de extrañar que las TIC se hayan ido integrando progresivamente en el ámbito educativo. Su integración comenzó en España alrededor de los años 2000 cuando las distintas Comunidades Autónomas optaban por elaborar programas o proyectos que, además de la formación del profesorado en el uso de herramientas tecnológicas en el aula, incluyeran una dotación masiva de recursos TIC para los centros.

De esta manera, se fue creando un alto interés por parte de los docentes en conocer cómo sacarle partido a todo ese material en el proceso de enseñanza-aprendizaje, es por ello que muchos profesores y profesoras han centrado gran parte de sus esfuerzos en formarse en el aprovechamiento de las TIC en las aulas. Este creciente interés ha hecho que, al mismo tiempo, las administraciones públicas centren algunos de sus medios en la elaboración de plataformas, portales y recursos didácticos digitales.

Tanto es así, que actualmente existen muchos portales institucionales. Plataformas que no solo sirven como repositorio, sino también como entornos virtuales que fomentan el intercambio de recursos y propuestas entre el profesorado, además de ser un sitio web al que acudir las familias y el alumnado para trabajar contenidos curriculares a través de un entorno atractivo.

Uno de los componentes más importantes de los portales educativos son la ingente cantidad de materiales didácticos que ofrecen para los diferentes usuarios que hacen uso del mismo. Esta cuestión tiene relación con el principal objetivo de este trabajo, que consiste en analizar las estrategias, políticas, etc., existentes con respecto a la producción de materiales didácticos digitales desde la perspectiva de diferentes responsables de portales institucionales.

\section{Plataformas y portales educativos}

Aunque el principal objetivo de este estudio no es una descripción de los diferentes portales institucionales existentes, se hace necesario realizar una revisión de las distintas características que ellos poseen.

Diferentes autores (Gértrudix, 2006; López Carreño, 2008; Santana, Eírin y Marín, 2017) han analizado y descrito las diferentes características de los portales educativos institucionales, tanto del estado español, como de las diferentes comunidades autónomas. Haciendo un resumen de los diferentes estudios encontrados podemos decir que: las plataformas y portales educativos son sitios web que se ponen a disposición de la comunidad educativa, ofertando servicios y contenidos que potencien el proceso de enseñanza-aprendizaje desde un entorno más llamativo para el alumnado. Por lo general, cuando hablamos de portales educativos visualizamos recursos interactivos donde alumnos y alumnas trabajan contenidos curriculares de manera lúdica. Sin embargo, este tipo de plataformas están destinadas a un uso didáctico, por ello, recopilan recursos educativos de todo tipo: interactivos, audiovisuales, textuales, etc.

Al mismo tiempo, debemos ser conscientes de que los portales educativos no son un mero repositorio de información, ya que también cumplen con una función de asesoramiento hacia el profesorado, contribuyen a la formación permanente de dicho colectivo y abren vías de comunicación entre los distintos agentes de la comunidad educativa. A continuación, enumeramos las características de estos sitios web, propuestas por Area (2011):

- Permiten el acceso a una gran cantidad de información.

- La información se representa de forma multimediada.

- El formato de organización y manipulación de la información es hipertextual.

- Permiten la publicación fácil y difusión de ideas y trabajos.

- Permiten la comunicación interpersonal tanto en tiempo real como diferido.

- Permiten la creación y gestión de espacios personalizables.

Además de las características recogidas, resulta importante señalar que Area (2003) propone una clasificación de sitios web educativos en función de sus finalidades, distinguiendo así entre sitios web informativos y sitios web formativos. Dentro de los primeros diferencia entre sitios web institucionales, y webs de recursos y bases de datos educativos. $Y$ dentro de los segundos, discierne entre entornos de teleformación e intranets educativas, y material didáctico web.

Las diferentes webs descritas por el autor, en el nuevo escenario que nos ocupa, suelen estar integradas en un mismo portal institucional, ofreciendo al usuario todos los servicios en un mismo paquete, es decir, tanto los recursos, materiales didácticos, etc., como el entorno social forman parte de un todo. 


\subsection{Iniciativas institucionales y privadas}

Tanto el estado, como la mayoría de las autonomías disponen, a través de cada Consejería de Educación, de su propio portal institucional. Estos portales tienen su origen en el compromiso de las Administraciones de integrar las TIC en las aulas, por lo que ponen a disposición de la comunidad educativa un catálogo que facilite el acceso a recursos digitales y favorezca dicha integración de las TIC en el día a día de la práctica docente. Es por ello que se caracterizan por ofrecer recursos cuyos contenidos están clasificados por etapas, cursos y asignaturas, facilitando así la localización de aquello que se está buscando. En la página de la revista Educación $3.0^{2}$ podemos acceder a cada uno de ellos y observar sus diferentes características.

También es importante destacar que hay una creciente tendencia a la participación de empresas privadas en este sector tecnológico-educativo. Casi la totalidad de las editoriales disponen de sus propias plataformas tecnológicas, donde han pasado del formato impreso de los libros de texto al formato digital, ofreciendo a su vez una serie de servicios que haga más llamativa la inversión en su empresa por parte de la comunidad educativa: entre otras cosas, proporcionan al profesorado las programaciones anuales, trimestrales y unidades didácticas asociadas a sus libros, incorporan algunas actividades interactivas para que dispongan de ellas en la pizarra digital, o permiten al profesorado crear los exámenes del tema escogiendo con libertad el número de preguntas y el tipo.

\section{Materiales didácticos digitales}

A pesar de haber comentado anteriormente la amplia variedad existente de portales educativos y sus tipos, el documento que nos ocupa estará centrado en analizar la visión de los responsables portales institucionales sobre la producción y creación de materiales didácticos digitales. Para ello, es necesario realizar una breve revisión de las investigaciones realizadas hasta este momento sobre el mismo.

Diferentes autores han realizado estudios sobre la conceptualización y la evolución que han tenido los materiales didácticos digitales (Cabero, 2001; Area, 2003; Cacheiro, 2011; Aguilar, Ayala, Lugo y Zarco, 2014; Castro, Rodríguez y Peirats, 2017; Area, 2017, entre otros).

Desde la aparición de la Web 2.0 (0`Reilly, 2005), la cantidad de recursos educativos digitales ha aumentado de manera exponencial durante los últimos años. Definir lo que es un material didáctico digital es una ardua tarea para aquellos que actualmente nos dedicamos a investigar sobre esta cuestión. Area (2017), en uno de sus últimos artículos elabora un inventario sobre ese conjunto de recursos educativos que se ofertan desde la red y que compartimos a continuación:

- Objeto digital: es un archivo digital;, por ejemplo, un documento, un vídeo, una fotografía, etc. Haciendo una unión de los mismos se puede construir un material didáctico.

- Objeto digital de aprendizaje: al contrario que el

\footnotetext{
2 Portales educativos españoles:

https://www.educaciontrespuntocero.com/recursos/portaleseducativos-comunidades-autonomas-espana/46781.html (Actualizado a 2 de mayo del 2017)
}

anterior, éste sí tiene una intencionalidad didáctica. Existen multitud de ellos a disposición del profesorado y el alumnado en las diferentes páginas del ciberespacio.

- Entorno didáctico digital: se trata de una estructura didáctica compuesta por diferentes objetos digitales y con una clara intencionalidad didáctica. Algunos ejemplos serían: una unidad didáctica, un espacio colaborativo para el profesorado y el alumnado, una lección, etc. Existe una cierta similitud con los llamados LMS.

- Libros de texto digital: se consideran la evolución de los textos escolares en papel. Incorporan recursos visuales, así como actividades a realizar por el alumnado. Suelen estar incorporados en plataformas en línea.

- Apps, herramientas y plataformas on-line: son herramientas que pueden ser creadas, en algunas ocasiones, con intencionalidad educativa. El auge de los dispositivos móviles en educación ha hecho que multitud de profesionales vean una oportunidad de negocio en este tipo de herramienta.

- Entornos inteligentes de aprendizaje: tienen relación con las analíticas de aprendizaje y el big data. Se trata de una recopilación de datos del alumnado que puedan ser manejados para mejorar la calidad de los materiales en relación a las necesidades del usuario.

- Materiales didácticos tangibles: tienen relación con la robótica educativa. Su incorporación a los centros escolares está tardando debido a su coste. Es una línea de trabajo en la que administraciones y empresas están trabajando.

- Materiales digitales para la docencia: no están creados para el alumnado, ya que su principal destinatario es el profesorado. Algunos ejemplos serían: blogs del profesorado, programaciones didácticas, experiencias de aula, etc.

Dicho inventario se corresponde con la multitud de materiales existentes en los diferentes portales educativos institucionales. Se hace necesario aclarar que la mayoría de estos recursos son alojados por los propios por los usuarios de dichas plataformas. Nuestro objetivo no es analizar la gran variedad de recursos disponibles, sino identificar aquellos materiales producidos por las instituciones: sus características, proceso de creación, autorías, desarrollo y evaluación de los mismos.

Aguilar, Ayala, Lugo y Zarco (2014) nos muestran algunos criterios de evaluación para los materiales didácticos que resumimos a continuación:

- Debe considerar el contexto de uso de los materiales, teniendo en cuenta las estrategias de enseñanza, las características del usuario, su nivel de interactividad, su presentación, etc.

- Debe haber distintos perfiles profesionales en la creación y evaluación de los materiales didácticos. Además, debe considerar la influencia en el aprendizaje del alumnado.

- La evaluación debe realizarse en distintos momentos del ciclo de vida de los materiales; puede ser tanto desde el momento del desarrollo como después de su uso. Se puede 
González Ruiz, Carlos José; Martín Gómez, Sebastián y Vega Navarro, Ana (2018). "Portales educativos: la producción de materiales didácticos digitales". @tic revista d'innovació educativa, 20, 89-97.

evaluar por dimensiones o componentes conceptuales.

\subsection{Políticas de producción de materiales didácticos digitales}

Si nos centramos en las políticas educativas con respecto en la producción y distribución de materiales didácticos digitales existen diferentes documentos que nos pueden ayudar a entender dicho proceso (Gértrudix, Álvarez, Galisteo, Gálvez, y Gértrudix, F., 2007; Area y Hernández, 2010; Mülbert y Lumi, 2011; Sanabria, Álvarez Núñez y Peirats, 2017).

Los citados autores realizan un recorrido sobre las políticas establecidas por el Ministerio en los últimos años en torno a los contenidos educativos digitales. Es de nuestro interés compartir un resumen de dichos trabajos y que tienen relación con lo expuesto en este trabajo. Para ello, hemos realizado un resumen de las líneas establecidas durante los últimos años y que todavía tienen vigencia a día de hoy.

- La creación y gestión de contenidos debe estar centrada en la experiencia del usuario y enfocada a la elaboración de objetos digitales de aprendizaje.

- Estándares y modelos de desarrollo homologables.

- Investigación para crear nuevos productos innovadores.

- Fomentar formación y ayuda al profesorado para que sea el propio creador de los contenidos.

Estamos de acuerdo con Sanabria, Álvarez y Peirats (2017) en que es necesario realizar investigaciones más amplias en este sentido y determinar con claridad cuál es la apuesta de la administración. En dicho artículo, en el apartado de resultados, podremos observar la visión que tienen los responsables institucionales con respecto a este tema.

\section{Metodología \\ 4.1 Objetivos}

Es importante hacer notar que este estudio parte del plan investigación del proyecto I+D EDU2015-64593-R: La escuela de la sociedad digital: análisis y propuestas para la producción y uso de los contenidos digitales educativos (Escuel@ Digit@l). En dicho estudio participan, las comunidades autónomas de Canarias, Valencia y Galicia; siendo coordinado por la comunidad autónoma de Canarias (Grupo EDULLAB, Universidad de La Laguna). Esta referencia no solo es vital para contextualizar este trabajo sino para comprender la estructura y decisiones tomadas en cuanto a la formulación de objetivos y metodología.

El problema de investigación que se aborda se centra en conocer cómo se organiza la producción de los materiales didácticos digitales de los portales institucionales, tomando como referencia la perspectiva de sus principales responsables. Es decir, nos centramos en conocer y aportar las consideraciones oportunas sobre cómo se lleva a cabo la fase de producción de estos materiales por parte del repositorio nacional Procomún (Red de Recursos Educativos en Abierto), del Ministerio de Educación, Cultura y Deporte (MECD); y del portal de recursos regional Ecoescuela 2.0 (Área de
Tecnología Educativa de la Consejería de Educación y Universidades del Gobierno de Canarias).

\subsubsection{Objetivo general}

Conocer el proceso de producción de los materiales didácticos digitales de los portales institucionales.

\subsubsection{Objetivos específicos}

- Describir la fase de producción de los materiales didácticos digitales de creación propia de los portales institucionales Procomún y Ecoescuela 2.0.

- Analizar las diferencias en la forma de producir los materiales didácticos digitales propios que siguen los portales institucionales Procomún y Ecoescuela 2.0.

\subsection{Método}

Debido a la naturaleza de los objetivos que se pretenden alcanzar en este estudio, se opta por una metodología de investigación cualitativa. Se entiende, por tanto, este estudio como una aproximación a la realidad de los casos seleccionados que tiene por propósito describir el fenómeno del que es objeto este trabajo, tomando como referencia a Gutiérrez (2017), el cual describe el patrón de la investigación cualitativa: selección del problema, formulación de interrogantes, recopilación de información, registro y análisis de la información (Spradley, 1980).

\subsection{Selección de la muestra}

Es preciso concretar que este estudio centra la atención en el caso de portales de recursos educativos institucionales, en concreto el repositorio nacional Procomún (Red de Recursos Educativos en Abierto), del Ministerio de Educación, Cultura y Deporte (MECD) y del portal regional Ecoescuela 2.0 (Área de Tecnología Educativa de la Consejería de Educación y Universidades del Gobierno de Canarias). Se escogen estos casos, al tratarse de dos portales educativos institucionales analizados en el proyecto de investigación anteriormente mencionado, siendo además dos organizaciones referentes en la creación de materiales y recursos didácticos de apoyo al profesorado, en sus respectivos ámbitos de alcance (a nivel nacional y a nivel de Canarias, respectivamente).

\subsection{Técnicas e instrumentos}

Para este estudio se diseña como instrumento de recogida de datos un guion de entrevista semiestructurada, que permite recoger la visión de los responsables de los portales educativos institucionales sobre la fase de producción de los materiales didácticos digitales de creación propia de cada repositorio. Este guion ha sido elaborado previamente para entrevistas destinadas a editores de portales educativos tanto privados como públicos de Educación Primaria de Canarias, Valencia y Galicia en el proyecto Escuel@ Digit@।. Se optan por las siguientes dimensiones, que organizan la información recogida:

- $\quad$ Organización del portal

- $\quad$ Creación de Materiales didácticos digitales

- $\quad$ Evaluación de Materiales didácticos digitales

- $\quad$ Modelo pedagógico

- Influencia económica

- $\quad$ Valoración 
González Ruiz, Carlos José; Martín Gómez, Sebastián y Vega Navarro, Ana (2018). "Portales educativos: la producción de materiales didácticos digitales". @tic revista d'innovació educativa, 20, 89-97.

El presente trabajo se concreta en la dimensión de creación de materiales didácticos digitales, correspondiéndose con el objetivo general y objetivos específicos descritos.

\subsubsection{Aplicación y procedimiento}

El procedimiento para la recogida de datos se desarrolló iniciando un contacto inicial con los responsables de los portales mencionados. En este primer acercamiento, se entregó documentación sobre el proyecto de investigación y se explicó sobre qué consistiría la entrevista, describiendo las dimensiones y tiempo estimado de la misma.

En el caso del portal Ecoescuela 2.0, se contactó con uno de los responsables del portal institucional y coordinador TIC de la Dirección General de Ordenación, Innovación y Promoción Educativa de la CEUCD del Gobierno de Canarias. La entrevista se realizó de manera presencial en las dependencias del Área de Tecnología Educativa- ATE [Medusa], situadas en el municipio de San Cristóbal de La Laguna, Santa Cruz de Tenerife). Se utilizó una grabadora externa de audio para registrar la información.

Para la entrevista a los responsables del portal nacional Procomún, se optó por una entrevista grupal, al conocer el interés de varios sujetos de participar en el estudio. El contacto se realizó con el director del Instituto Nacional de Tecnologías Educativas y formación del Profesorado (INTEF); con la jefa de Área de Recursos Educativos Digitales del mismo organismo; y el director del Centro Nacional de Desarrollo Curricular en Sistemas no Propietarios (CeDeC). En este caso, la entrevista se realizó de forma on-line a través de la herramienta que nos ofrece Google para videoconferencia: Hang-Out.

Una vez recogidos los datos, se transcribió la información y se procedió a su codificación y categorización, según las dimensiones de análisis establecidas previamente. Se concluyó con la elaboración de matrices de doble entrada en la que identifica horizontalmente las dimensiones o categorías de análisis, y verticalmente a los sujetos, para sintetizar las ideas expresadas por el entrevistado con relación a cada una de las categorías. En este sentido, se establecieron dos niveles de análisis, siendo en el segundo nivel cuando se comparan las visiones de los responsables de ambos portales en referencia a la producción de materiales didácticos digitales.

\section{Resultados}

Una vez llevado a cabo el proceso de análisis, se presentan los resultados más relevantes sobre el proceso de creación de materiales didácticos digitales de los dos portales institucionales de recursos en el que se centra este trabajo.

\subsection{Análisis comparativo}

Se presentan los resultados del análisis de los datos obtenidos en las entrevistas realizadas a los responsables de ambos portales. Estas entrevistas semiestructuradas están organizadas en diferentes categorías. En este caso, centraremos la atención en la categoría que hace referencia a la creación de materiales desde dichos repositorios. En el instrumento de recogida de datos se plantean cuestiones como: ¿quiénes son los autores de los materiales publicados en el portal?; ¿qué perfiles profesionales tienen?; ¿existen equipos de trabajo interdisciplinares para la creación de los materiales didácticos digitales que ofrece el portal?; ¿qué criterios han seguido para la creación o selección de los materiales?; ¿cuál es el proceso de creación de los materiales digitales incorporados al portal?

A continuación, se presentan las principales aportaciones de los responsables entrevistados de cada portal institucional (Tabla 1).

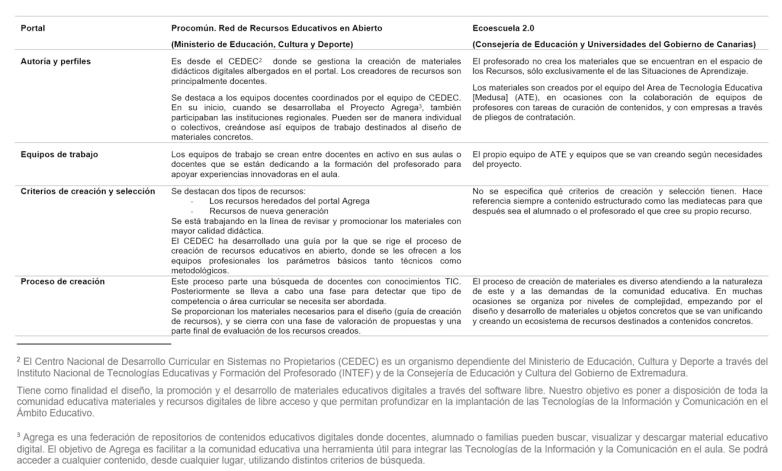

Tabla 1. Análisis comparativo de la creación de materiales digitales de los portales institucionales (ver ampliación en Anexo I)

\subsubsection{Autoría los recursos educativos disponibles en los portales}

En referencia a las dimensiones relacionada con la autoría y perfil de los creadores de materiales, vemos similitudes en ambos portales. La producción de los recursos se lleva a cabo desde organismos relacionados con la gestión de los sitios web. Cabe destacar que los portales tienen una característica que los diferencia: la subida de materiales libre por parte de docentes. En portal Ecoescuela 2.0 no está concebido para que los docentes puedan compartir en la plataforma sus creaciones; mientras que esto si es posible en el portal propiedad del Ministerio de Educación. Por su parte, los materiales albergados en Procomún son desarrollados por el Centro Nacional de Desarrollo Curricular en Sistemas no Propietarios (CEDEC). En el portal de la Comunidad Autónoma de Canarias, es la propia Area de Tecnología Educativa [Medusa] (ATE) quien desarrolla esta tarea.

La forma de operar difiere en este caso, optando ambas plataformas por perfiles profesionales distintos. En el primer caso se plantea la producción a equipos docentes propios del CEDEC, dedicados especialmente a generar contenidos. En el portal Ecoescuela 2.0 la producción de materiales la realiza su propio equipo de profesionales, dependiendo del tipo de producción y naturaleza de los materiales, si se llevan a cabo colaboraciones con docentes en activo, o empresas dedicadas a la producción de recursos.

\subsubsection{Criterios para el diseño y desarrollo de los materiales}

La información obtenida en relación con los criterios seguidos para el diseño de los materiales por parte de los gestores de ambas plataformas es escasa y poco aclaratoria.

En el caso del portal Procomún, se centra la atención en diferenciar dos grandes tipos de materiales existentes en su plataforma: aquellos que originalmente se crearon para ser incluidos en el repositorio Agrega y los más recientes, creados después de la reorganización del 
portal. Se sostiene que actualmente se está tratando de escoger para su promoción aquellos materiales con más calidad tanto técnica como pedagógica. Se extrae, por tanto, que la calidad de los objetos albergados es un criterio presente a la hora de, no solo crear materiales, sino de albergar recursos propios de docentes externos. Al mismo tiempo, el proceso de creación de materiales sí cuenta con unos criterios y pautas de producción concretas, que, aunque no descritas en la entrevista, se tienen presentes cuando los equipos creadores asumen la tarea de diseñar materiales concretos.

Las aportaciones de los responsables del portal Ecoescuela 2.0 también generan dudas. No obstante, se hace alusión a un tipo de materiales de nueva generación que son creados con la intención de que los docentes interesados puedan crear sus propios recursos. Esto se traduce en objetos digitales, los cuales pueden ser modificados o usados por los profesionales a conveniencia, adaptándolos a la realidad de su práctica educativa. Esto puede ser un punto diferencial frente a otros portales que ofrecen recursos empaquetados que no permiten su modificación según las necesidades del docente.

\subsubsection{Proceso de diseño y desarrollo de materiales}

En último lugar, observando lo aportado por los responsables entrevistados en cuanto a la forma de abordar el proceso de creación de materiales, se puede detectar como el CECED se rige por un proceso y ritmo de trabajo organizado y sistematizado. De esta forma, los materiales elaborados por sus equipos y que están alojados en el portal Procomún siguen la siguiente estructura en la cadena de producción:

1. Organización de equipos de trabajo, compuestos principalmente por docentes especializados en el trabajo con TIC en el aula.

2. Análisis de necesidades. Detección de las competencias, áreas o contenidos que necesitan ser abordados

3. Dotación de los recursos necesarios a los equipos docentes, especialmente de la guía de creación de recursos en abierto

4. Presentación de propuestas por parte de los docentes y valoración

5. Evaluación de los materiales seleccionados

Se aprecia una estructura ordenada y lógica en el proceso de creación de los materiales. Si bien no se especifica cómo se evalúan los materiales propuestos, se puede intuir que pasan por varias fases evaluativas, entre ellas "pruebas piloto" con grupos reducidos de alumnado.

Desde el portal gestionado por el ATE, por su parte, no se aporta información sobre el proceso habitual a desarrollar cuando se crea un material digital educativo. Esto puede ser debido a la variedad de situaciones y razones presentes en el proceso cuando se inicia esta tarea. Sin embargo, se comenta que en la producción de recursos se parte de objetos y contenidos simples, que luego se van agrupando en materiales más completos. De esta forma, entendemos que su forma de operar en este sentido no es lineal sino escalonada, con el fin de crear ecosistemas o paquetes de materiales amplios compuestos por diferentes piezas que compongan un material estructurado o una colección de estos.

\section{Conclusiones}

Durante el desarrollo del artículo hemos podido comprobar la importancia que le confieren todas las instituciones a la integración de las tecnologías de la información y la comunicación. El marco europeo presentado, así como las distintas iniciativas realizadas desde el ámbito nacional y autonómico hacen que nos encontremos ante un contexto que se seguirá desarrollando en los próximos años. Esto exige una implicación y colaboración por parte de todos los agentes (empresas, universidades, centros educativos, instituciones, etc.) que forman parte de este proceso imparable.

Los portales educativos están llenos de materiales didácticos. La aparición de la Web 2.0 ha cambiado el escenario de los recursos a disposición de los agentes educativos. La posibilidad de que sea el propio usuario el que suba los materiales a los portales educativos hace necesaria una nueva clasificación (Area, 2017) y distinción de los materiales disponibles en las diferentes plataformas. Además, debido a la gran cantidad de herramientas disponibles, hemos podido observar cómo la producción de dichos materiales puede ser también creación del propio profesorado, cuestión de especial importancia, pues son ellos los encargados de valorar su utilidad en el aula.

La autoría de los materiales es una cuestión a analizar durante los próximos años. Mientras que algunas instituciones apuestan por los propios docentes (INTEF), hay otras instituciones que apuestan por diferentes empresas para la creación de los mismos (Eco-Escuela 2.0). Se hace necesaria una colaboración constante entre el profesorado que pondrá en práctica dichos materiales y el equipo de producción de los mismos, para de esta manera asegurarnos un material de calidad.

Con respecto a los criterios que han de guiar el proceso de creación de los materiales didácticos digitales nos encontramos que las actuaciones de la administración en torno a la creación y gestión de contenidos suelen estar centradas en el usuario y enfocadas hacia la elaboración de objetos digitales de aprendizaje, cuestión que apuntaban ya hace unos años Gértrudix, Álvarez, Galisteo, Gálvez y Gértrudix (2007). Otra de las cuestiones que se observan es que en la creación y evaluación de materiales didácticos forman parte distintos perfiles de profesionales, y que se corresponde con una de los criterios de calidad establecidos por Aguilar, Ayala, Lugo y Zarco (2014).

Otra de las cuestiones clave, en este proceso es la formación del profesorado. Parece indicar, a la vista de los resultados obtenidos, que los dos portales aquí analizados, están empezando a tomar en cuenta a los mismos en la creación de los distintos materiales didácticos digitales. La apuesta emprendida por el CEDEC, en la que es el profesorado activo quien se encarga de su elaboración, es una de las líneas más importantes que hemos encontrado en dicho trabajo y que se corresponde con una de las últimas investigaciones realizadas con respecto a este sentido (Sanabria, Álvarez Núñez y Peirats, 2017). Sin embargo, una cuestión es que se les tome en cuenta para la creación de los mismos y otra muy distinta el hecho de que se ofrezca una formación en torno a esta temática. Las administraciones no pueden ni deben darle la espalda al colectivo que está a pie de obra, ya que son los que en última instancia usarán dichos materiales en los procesos de enseñanza-aprendizaje. 
González Ruiz, Carlos José; Martín Gómez, Sebastián y Vega Navarro, Ana (2018). "Portales educativos: la producción de materiales didácticos digitales". @tic revista d'innovació educativa, 20, 89-97.

En definitiva, podemos afirmar que la producción de materiales educativos digitales se encuentra en un estado un poco difuso debido a la gran cantidad de recursos que disponemos a nuestra disposición. Una de las razones es la llamada Web 2.0, la cual ha modificado el rol del profesorado, ya que no solamente es consumidor de los materiales ofrecidos por los diferentes portales, sino que también forma parte en la creación de los mismos, tanto de forma individual como de forma colectiva, y que hemos podido comprobar en el análisis realizado en este estudio.

\section{Bibliografía}

Aguilar, I., Ayala, J., L, O., y Zarzco, A. (2014). Análisis de criterios de evaluación para la calidad de los materiales didácticos digitales. Revista iberoamericana de ciencia tecnología y sociedad, 9(25), 73-89. Recuperado de: http://ir.uv.es/OJuZnru

Area, M. (2006). Veinte años de políticas institucionales para incorporar las tecnologías de la información y la comunicación al sistema escolar. En J. Sancho (coord.), Tecnologías para transformar la educación. Madrid: Akal.

Area, M. (2011). Los efectos del modelo 1:1 en el cambio educativo en las escuelas. Evidencias y desafíos de las políticas iberoamericanas. Revista IberoAmericana de Educaçao, 56, 49-74. Recuperado de: http://ir.uv.es/IF14wyR

Area, M. (2017). La metamorfosis digital del material didáctico tras el paréntesis Gutenberg. Relatec, 16(2), 13-28. https://doi.org/10.17398/1695288X.16.2.13

Area, M. y Hernández, V. (2010). La producción de material educativo multimedia. Tendencias pedagógicas, 16, 65-88. Recuperado de: http://ir.uv.es/AKETmPO

Area, M. (2003). De los web educativos al material educativo web, Comunicación y Pedagogía, 188, 3238.

Cabero, J. (2001). Tecnología educativa: diseño y utilización de medios en la enseñanza. Barcelona: Paidós.

Cacheiro, M.L. (2011). Recursos educativos TIC de información, colaboración y aprendizaje. Pixel-Bit. Revista de Medios y Educación, 39, 69-81. Recuperado en http://www.redalyc.org/pdf/368/36818685007.pdf

Castro, M.; Rodríguez, J. y Peirats, J. (2017). Materiales Didácticos, Libros de Texto y Educación Inclusiva. Educatio siglo XX, 35 (3), 11-16. Recuperado de: http://ir.uv.es/UFplPkO

Colás, P. (2015). La evaluación de las políticas educativas TIC: enfoques y metodologías. En J. De Pablos (coord.), Los centros educativos ante el desafío de las tecnologías digitales. Madrid: La Muralla.

De Pablos, J., Colás, P. y Villarciervo, P. (2010). Políticas educativas, buenas prácticas y TIC en la comunidad autónoma andaluza. Revista Electrónica Teoría de la Educación: Educación y Cultura en la Sociedad de la Información, 11(1), 180-202. Recuperado de: http://gredos.usal.es/jspui/bitstream/10366/7283 8/1/Monografico_completo.pdf

Domínguez Romero, E., Fernández-Pampillón Cesteros, A., y Armas Ranero, I. de. (2011). Herramienta para la revisión de la Calidad de Objetos de Aprendizaje Universitarios(COdA): guía del usuario. v.1.1.Recuperado de: http://eprints.ucm.es/12533/

Gértrudix, M.; Álvarez, S.; Galisteo, A.; Gálvez, M.C. y Gértrudix, F. (2007). Acciones de diseño y desarrollo de objetos educativos digitales: programas institucionales. RUSC,4(1). Recuperado de: http://www.redalyc.org/html/780/78040107

González Ruiz, C.J. (2016). Evaluación de la implementación del programa REDXXI/Escuela 2.0. Universidad de Salamanca, España

Gutiérrez, L. (2017). Paradigmas cuantitativo y cualitativo en la investigación socio-educativa: proyección y reflexiones. Paradigma, 14(1y2), 7-25. Recuperado de: http://ir.uv.es/NuM5nkl

Kozma, R. (2005). National policies that connect ICTBased education reform to economic and social development. Human Technology, 1(2), 117-156. Recuperado de: http://ir.uv.es/ejqpBoO

Marín Suelves, D.; Granados, J.; Morote, D. y Peirats, J. (2017). Descripción y análisis de la plataforma institucional Mestre a casa. En A. Gutiérrez Martín, A. García Matilla y R. Collado Alonso (eds.). Actas del III Congreso Internacional de Educación Mediática y Competencia Digital, 2015-2020. Valladolid: Facultad de Educación y Facultad de Ciencias Sociales, Jurídicas y de la Comunicaciónn, Universidad de Valladolid.

Martínez Figueira, Ma.E. (2006). Políticas autonómicas para la integración de las TIC en centros educativos, Revista Latinoamericana de Tecnología Educativa, .5(2), 97-112. Recuperado de: https://relatec.unex.es/article/view/229

Mülbert, A. y Lumi, F. (2011). La transformación del proceso de diseño instruccional: del impreso a la multimedia. Revista iberoamericana de educación a distancia, .14 (1), 41-54. Recuperado de: http://ir.uv.es/4vMuRwl

O'Reilly, T. (2005). What is WEB 2.0. Recuperado de: http://www.oreilly.com/pub/a/web2/archive/what-isweb-20.html

Sanabria, A., Álvarez Nuñez, Q. y Peirats, J. (2017). Las políticas educativas en la producción y distribución de materiales didácticos digitales. Relatec, 16 (2), 63-77. $288 \times .16 .2 .63$

Spradley, J. (1980) Observación Participante, New York: Rinehart and Winston.

Valverde, J., Garrido, M., y Sosa, M.J. (2010). Políticas educativas para la integración de las TIC en Extremadura y sus efectos sobre la innovación didáctica y el proceso enseñanza-aprendizaje: la percepción del profesorado. Revista de Educación (352), 99-124. Recuperado de: http://ir.uv.es/h5CczNs 
González Ruiz, Carlos José; Martín Gómez, Sebastián y Vega Navarro, Ana (2018). "Portales educativos: la producción de materiales didácticos digitales". @tic revista d'innovació educativa, 20, 89-97.

I Cita recomendada de este artículo

González Ruiz, Carlos José; Martín Gómez, Sebastián y Vega Navarro, Ana (2018). "Portales educativos: la producción de materiales didácticos digitales". @tic revista d'innovació educativa, 20-89-97.

\section{ANEXO I}

Tabla 1. Análisis comparativo de la creación de materiales digitales de los portales institucionales

$\begin{array}{ll}\text { Portal } & \begin{array}{l}\text { Procomún. Red de Recursos Educativos en } \\ \text { Abierto }\end{array} \\ \begin{array}{l}\text { (Ministerio de Educación, Cultura y yela } 2.0 \\ \text { Deporte) }\end{array} & \begin{array}{l}\text { (Consejería de Educación y Universidades } \\ \text { del Gobierno de Canarias) }\end{array}\end{array}$

$\begin{array}{ll}\text { Autoría y } & \text { Es desde el CEDEC3 donde se gestiona la } \\ \text { perfiles } & \text { creación de materiales didácticos digitales } \\ & \text { albergados en el portal. Los creadores de } \\ \text { recursos son principalmente docentes. }\end{array}$

Se destaca a los equipos docentes coordinados por el equipo de CEDEC. En su inicio, cuando se desarrollaba el Proyecto Agrega 4 , también participaban las instituciones regionales. Pueden ser de manera individual o colectivos, creándose así equipos de trabajo destinados al diseño de materiales concretos.

\begin{abstract}
El profesorado no crea los materiales que se encuentran en el espacio de los Recursos, sólo exclusivamente el de las Situaciones de Aprendizaje.

Los materiales son creados por el equipo del Area de Tecnología Educativa [Medusa] (ATE), en ocasiones con la colaboración de equipos de profesores con tareas de curación de contenidos, y con empresas a través de pliegos de contratación.
\end{abstract}

\section{Equipos de Los equipos de trabajo se crean entre trabajo docentes en activo en sus aulas o docentes que se están dedicando a la formación del profesorado para apoyar experiencias innovadoras en el aula.}

El propio equipo de ATE y equipos que se van creando según necesidades del proyecto.

Criterios de
creación y
selección
selección
Se destacan dos tipos de recursos:

- Los recursos heredados portal Agrega

- Recursos de nueva generación

Se está trabajando en la línea de revisar y promocionar los materiales con mayor calidad didáctica.

EI CEDEC ha desarrollado una guía por la que se rige el proceso de creación de recursos educativos en abierto, donde se les ofrecen a los equipos profesionales los parámetros básicos tanto técnicos como metodológicos.
No se especifica qué criterios de creación y el selección tienen. Hace referencia siempre a contenido estructurado como las mediatecas para que después sea el alumnado o el profesorado el que cree su propio recurso.

\footnotetext{
${ }^{3}$ El Centro Nacional de Desarrollo Curricular en Sistemas no Propietarios (CEDEC) es un organismo dependiente del Ministerio de Educación, Cultura y Deporte a través del Instituto Nacional de Tecnologías Educativas y Formación del Profesorado (INTEF) y de la Consejería de Educación y Cultura del Gobierno de Extremadura. Tiene como finalidad el diseño, la promoción y el desarrollo de materiales educativos digitales a través del software libre. Nuestro objetivo es poner a disposición de toda la comunidad educativa materiales y recursos digitales de libre acceso y que permitan profundizar en la implantación de las Tecnologías de la Información y Comunicación en el Ámbito Educativo.

${ }^{4}$ Agrega es una federación de repositorios de contenidos educativos digitales donde docentes, alumnado o familias pueden buscar, visualizar y descargar material educativo digital. El objetivo de Agrega es facilitar a la comunidad educativa una herramienta útil para integrar las Tecnologías de la Información y la Comunicación en el aula. Se podrá acceder a cualquier contenido, desde cualquier lugar, utilizando distintos criterios de búsqueda.
} 
González Ruiz, Carlos José; Martín Gómez, Sebastián y Vega Navarro, Ana (2018). "Portales educativos: la producción de materiales didácticos digitales". @tic revista d'innovació educativa, 20, 89-97.

Proceso de creación
Este proceso parte una búsqueda de docentes con conocimientos TIC. Posteriormente se lleva a cabo una fase para detectar que tipo de competencia o área curricular se necesita ser abordada. Se proporcionan los materiales necesarios para el diseño (guía de creación de recursos), y se cierra con una fase de valoración de propuestas y una parte final de evaluación de los recursos creados.
Este proceso de creación de materiales es diverso atendiendo a la naturaleza de este y a las demandas de la comunidad educativa. En muchas ocasiones se organiza por niveles de complejidad, empezando por el diseño y desarrollo de materiales $u$ objetos concretos que se van unificando y creando un ecosistema de recursos destinados a contenidos concretos. 